\begin{tabular}{|c|c|}
\hline Title & Quantitative phase field modeling for two-phase solidification process involving diffusion in the solid \\
\hline Author(s) & Ohno, Munekazu; Matsuura, Kiyotaka \\
\hline Citation & $\begin{array}{l}\text { A cta Materialia, 58(17), 5749-5758 } \\
\text { https://doi.org/10.1016j. actamat.2010.06.050 }\end{array}$ \\
\hline Issue Date & $2010-10$ \\
\hline Doc URL & http:/hdl.handle.net/2115/44154 \\
\hline Type & article (author version) \\
\hline File Information & A M58-17_5749-5758.pdf \\
\hline
\end{tabular}

Instructions for use 


\title{
Quantitative phase-field modeling for two-phase solidification process involving diffusion in the solid
}

\author{
Munekazu Ohno and Kiyotaka Matsuura
}

Division of Materials Science and Engineering, Faculty of Engineering, Hokkaido University, Kita 13 Nishi 8, Kita-ku, Sapporo, Hokkaido, 060-8628, Japan

\begin{abstract}
A quantitative phase-field model for two-phase solidification process is developed based on the antitrapping current approach with the free energy functional formulated to suppress the formation of an extra phase at the interface. This model appropriately recovers the free boundary problem for the motion of interface in the thin-interface limit and, importantly, it is applicable to the solidification process in binary alloy systems with arbitrary values of the solid diffusivities and interfacial energies. The performance of the present model is investigated for the peritectic reaction process in carbon steel. The present model exhibits excellent convergence behavior with respect to the interface thickness.
\end{abstract}

Keywords: Phase-field models; Peritectic solidification; Simulation 


\section{Introduction}

The description of microstructures during multi-phase solidifications is one of the most important issues in materials science. The phase-field model has been emerging as a powerful model to describe a variety of microstructural evolution processes [1-3]. The diffuse interface concept in this model allows us to describe the complex morphology of the microstructure without explicitly tracking the position of the interface. Of particular relevance to the present study is the development of multi-phase-field models for multi-phase solidification processes [3-9].

The motion of solid-liquid interface has been an issue tackled in the framework of free boundary problems (FBP). Basically, the solution of the phase-field simulation must be consistent with the FBP. However, the conventional models involve several anomalous interface effects [10]. Consequently, the outcome is not consistent with the FBP and is dependent on the interface thickness, $W$. A seminal scheme was devised to lift this problem based on the thin-interface limit $[11,12]$. For the single phase solidification in binary alloy systems, the FBP can be recovered with a finite value of $W$ by introducing an additional solute current, called the antitrapping current in the diffusion equation $[12,13]$. This model is called the quantitative phase-field model by which one can obtain $W$-independent and quantitatively accurate result under a given set of physical parameters. This quantitative phase-field model was applied to the analyses of several solidification phenomena [14-18] and it was recently 
extended to the description for the multi-component alloy [19]. Importantly, a quantitative phase-field model for two-phase solidifications was developed on the basis of the antitrapping current scheme [20]. However, there is a limitation in the application of these models. These models are applicable only to the alloy system without the solid diffusion (one-sided model). Hence, one cannot reproduce the equilibrium solidification during extremely slow cooling process and, instead, one inevitably describes the Scheil-type solidification process. Furthermore, it is not possible to appropriately deal with the peritectic reaction and transformation which accompanies the motion of the solid-solid interface controlled by the solid diffusion.

Based on an asymptotic analysis, the present authors recently extended the antitrapping current approach to the single phase solidification process involving diffusion in the solid, viz., the case for an arbitrary value of the solid diffusivity [21]. The computations for the dendrite growth demonstrated fairly reasonable convergence behavior of the outcomes such as the dendrite tip velocity, dendrite tip radius and concentration profile with respect to $W$. In the present study, we develop a model for two-phase solidification processes based on this antitrapping current approach. As mentioned above, the quantitative phase-field modeling was previously carried out for the two-phase solidification process [20]. Two notable improvements were achieved in that modeling; one is the elimination of a problem associated with an extra phase in the interface, which is involved in the conventional models as detailed 
later, and the other is the correction of the solute concentration field by introducing the antitrapping current. The former improvement associated with the phase field profile is indispensable for the antitrapping current to work successfully in the latter improvement. As mentioned above, however, this model cannot handle the diffusion in the solid. In addition, this model is actually validated only for the system in which all two-phase interfaces possess the same interfacial energy. These are the stringent limitations in the application.

In this study, we develop the quantitative phase-field model for the two-phase solidification in binary alloy systems with arbitrary values of the solid diffusivities and interfacial energies. For this, the antitrapping current approach of Ref. [21] is extended to the case for the two-phase solidification. Also, an appropriate free energy functional is devised to remove the problem related to the extra phase at the interface. We demonstrate the performance of the present model, focusing on the peritectic reaction in carbon steel.

\section{Modeling}

\subsection{Free energy functional}

In a binary alloy system, three phases exist during the eutectic or peritectic reaction, liquid and two solid phases. We employ three phase fields, $p_{i}$, indicating the existence of $i$ phase. The subscript $i$ with $i=1,2$ and 3 specifies the type of the phase. For generality in the discussion, also, we use indices $i, j$ and $k$ to distinguish the three phases. $p_{i}$ is allowed to vary 
from 0 to 1 and the following constraint is subjected to these phase fields,

$$
\sum_{i} p_{i}=1
$$

Then, for instance, the existence of $i$ phase is represented by the relation $\left(p_{i}, p_{j}, p_{k}\right)=(1,0,0)$.

The $j-k$ interface is characterized by the spatial profile of the phase fields in which $p_{j}$ and $p_{k}$

smoothly vary from 1 to 0 across the interface, whereas $p_{i}$ is always zero. Provided that this

spatial profile is realized, the conventional multi-phase-field model is formulated to become

identical to the model for the single phase solidification, which is the essential strategy of the

modeling. As discussed in the early studies [20, 22], however, the conventional models

involve the unexpected formation of an extra phase in the interface, More specifically, in

practice, $p_{i}$ is not zero but takes a finite value inside the $j-k$ interface region. In the description

of the motion of two-phase interface, hence, the multi-phase-field model is not consistent with

the model for the single-phase solidification. As shown later, moreover, the antitrapping

current approach does not work adequately when the unexpected extra phase exists, since the

antitrapping current term depends on the spatial profile of the phase fields. This problem

makes it impossible to perform quantitatively (and sometimes even qualitatively) accurate

simulation for the multi-phase solidifications.

In order to eliminate the extra phase, viz., in order for $p_{i}$ to be zero across the $j-k$ interface, one must satisfy the condition, $\lim _{p_{i} \rightarrow 0} \partial p_{i} / \partial t=0$, where $t$ is the time. Within the phase-field model, the time evolution of the phase field is described based on the free energy 
functional of the system, $F$, as written by the following time-dependent Ginzburg-Landau equation [20],

$$
\frac{\partial p_{i}}{\partial t}=-\left.M\left(\left\{p_{j}\right\}\right) \frac{\delta F}{\delta p_{i}}\right|_{\sum p_{j}=1},
$$

where $M\left(\left\{p_{j}\right\}\right)$ is the mobility which depends on the phase fields. The functional derivative of $F$ on the right-hand side of eq.(2) is evaluated with imposing the constraint of eq.(1). Using a Lagrange multiplier, the variational derivative in eq.(2) is expressed as,

$$
\left.\frac{\delta F}{\delta p_{i}}\right|_{\sum p_{j}=1}=\frac{\delta F}{\delta p_{i}}-\frac{1}{3} \sum_{j} \frac{\delta F}{\delta p_{j}},
$$

where the functional derivatives on the right-hand side are taken as if all the phase fields were independent. Therefore, the condition for the elimination of the extra phase can be rewritten as,

$$
\left.\frac{\delta F}{\delta p_{i}}\right|_{\sum p_{j}=1}=0 \quad \text { at } \quad p_{i}=0 \quad \forall \quad i .
$$

Specifically, the free energy functional $F$ should be formulated to satisfy the requirement that the only steady state solution of $p_{i}$ profile across the $j-k$ interface is $p_{i}=0$. Also, there is another requirement that $p_{i}=1$ is the stable solution in the bulk state of any phase $i$. Furthermore, the stability of these solutions against a fluctuation in the phase filed profiles should be guaranteed by the condition that the second derivative of free energy is always positive at $p_{i}=0$ and 1 , as detailed in Ref. [20].

The condition given by eq.(4) was not taken into account in the conventional 
multi-phase-field modeling. The so-called double obstacle potential with a higher order term can suppress the formation of the extra phase [22]. However, such a model is not pertinently mapped onto the thin-interface limit model. In the pioneering study on the quantitative phase-field modeling for two-phase solidifications [20], the existence of the extra phase was successfully suppressed by modifying the form of the free energy functional. However, the model presented in Ref. [22] is validated only for the system with the equal interfacial energies. Although a scheme for the description of unequal interfacial energies was proposed [20], it necessitates a revision in the available thin-interface limit analysis. No multi-phasefield model is currently developed for the system with unequal interfacial energies so as to ensure the consistency with the available quantitative phase-field model for the single phase solidification. In this section, we demonstrate the quantitative phase-field model, which is free from the problem with the extra phase in the interface and is consistent with the thin-interface limit model, for the two-phase solidification in the binary alloy system with arbitrary values of the solid diffusivities and interfacial energies. The crystalline anisotropy of the interfacial energy is not taken into account. However, the introduction of the anisotropy does not alter the essential parts in the modeling.

In this study, we propose the following form of the Ginzburg-Landau-type free energy functional,

$$
F=\int\left(\frac{\varepsilon^{2}}{4} \sum_{i}\left(\nabla p_{i}\right)^{2}+\omega\left(\left\{p_{i}\right\}\right)\left[\sum_{i} p_{i}^{2}\left(1-p_{i}\right)^{2}\right]+\sum_{i} g_{i}\left(\left\{p_{i}\right\}\right) f_{i}^{0}\left(c_{i}, T\right)\right) \cdot d V
$$


where $V$ is the unit volume. The details of each term in the integrand of eq.(5) are discussed below.

The first term in the integrand of eq.(5) represents the gradient energy term and $\varepsilon$ is the gradient energy coefficient. It is noted that the gradient energy coefficient does not depend on the type of the two-phase interface. This is in contrast to the fact that different gradient energy coefficients are used for the different interfaces as expressed by $\varepsilon_{i j}$ in the conventional models [4-9]. The form in eq.(5) is required to satisfy the condition given by eq. (4) [20]. The unequal interfacial energies are considered in the second term in eq.(5) as detailed below.

The second term in eq.(5), denoted here as $f_{\text {pot, }}$, represents the potential barrier between the bulk phases, which assures that the solidification is the first order transition. The term in the square bracket represents the sum of double well potentials, $p_{i}^{2}\left(1-p_{i}\right)^{2} . \omega\left(\left\{p_{i}\right\}\right)$ determines the potential height. In the conventional models [4-9], different constant values are assigned to the potential height for each interface, as expressed by $\omega_{i j}$, to describe the unequal interfacial energies. However, the condition, eq.(4), cannot be satisfied in such an approach. If $\omega$ is taken to be a constant in eq.(5), our free energy model is essentially equivalent to the one proposed in the early work [20] for the case of the equal interfacial energies. In their work [20], in order to handle the unequal interfacial energies, the higher order term in $p_{i}$ is added to the double well potential. However, such a model is not exactly reduced to the available thin-interface limit model. To deal with the unequal interface energies, in the present study, 
we introduce the $p_{i}$ dependence of $\omega$. In order to hold the condition given by eq. (4), $\omega\left(\left\{p_{i}\right\}\right)$ has to satisfy the following relation,

$$
\partial \omega\left(\left\{p_{i}\right\}\right) / \partial p_{j}=0 \text { at } p_{k}=0,1 \quad \forall j, \quad k
$$

The unequal interfacial energies are introduced by holding the following relation,

$$
\omega\left(\left\{p_{i}\right\}\right)=\frac{1}{2} \omega_{i j} \text { at } p_{k}=0
$$

where $\omega_{i j}$ denotes the potential height between $i$ and $j$ phases. In this study, we define $\omega\left(\left\{p_{i}\right\}\right)$ as follows,

$$
\omega\left(\left\{p_{i}\right\}\right)=\left(2 \sum_{i, j>i} p_{i}^{2} p_{j}^{2}\right)^{-1}\left(\sum_{i, j>i} \omega_{i j} \cdot p_{i}^{2} p_{j}^{2}+\omega_{t r i} \cdot\left(p_{i} p_{j} p_{k}\right)^{n}\right),
$$

with $n>1$. Here, $\omega_{\text {tri }}$ is a constant related to the potential height for the mixture state of three phases. It is trivial to check that $\omega\left(\left\{p_{i}\right\}\right)$ in eq.(8) satisfies eqs.(6) and (7). Note that $f_{\text {pot }}$ is exactly reduced to the corresponding term in the available thin-interface limit model for the single phase solidification [21] at $p_{k}=0$. The function $\omega\left(\left\{p_{i}\right\}\right)$ exhibits a discontinuity at $p_{k}=1$ as $\lim _{p_{k} \rightarrow 1} \omega\left(\left\{p_{i}\right\}\right)=(1 / 2)\left(\omega_{j k} p_{j}^{2}+\omega_{k i} p_{i}^{2}\right) /\left(p_{j}^{2}+p_{i}^{2}\right)$. However, the discontinuity vanishes in $f_{\text {pot }}$ and there is no problem in the numerical simulations. In eq.(8), the term multiplied by $\omega_{t r i}$ plays an auxiliary role in raising the potential height for the mixture state of three phases, of which the contribution is determined by $\omega_{t r i}$ and $n$. Our preliminary calculations showed that this term is quite useful in suppressing the formation of the extra phase when the difference between the interfacial energies is large. It should be stressed that the unequal interfacial energies are considered in $f_{\text {pot }}$ which satisfies the condition of eq.(4) and keeps the consistency 
with the available quantitative phase-field model.

The third term in the integrand of eq.(5) represents the contribution of the free energies of the bulk phases, yielding the driving force for the solidification. $f_{i}^{0}\left(c_{i}, T\right)$ is the free energy density of $i$ phase given as a function of the concentration of $i$ phase, $c_{i}$, and temperature $T . g_{i}\left(\left\{p_{i}\right\}\right)$ is the interpolating function and we adopt the following form devised in the early work [20],

$$
g_{i}\left(\left\{p_{i}\right\}\right)=\frac{p_{i}^{2}}{4}\left\{15\left(1-p_{i}\right)\left[1+p_{i}-\left(p_{k}-p_{j}\right)^{2}\right]+p_{i}\left(9 p_{i}^{2}-5\right)\right\}
$$

This function $g_{i}\left(\left\{p_{i}\right\}\right)$ reduces to the polynomial $\widetilde{g}\left(p_{i}\right)=p_{i}{ }^{3}\left(10-15 p_{i}+6 p_{i}{ }^{2}\right)$ on $i-j$ and $i-k$ interfaces when the normalization condition eq.(1) is imposed [20].

As mentioned above, the free energy functional proposed in this study is formulated to remove the extra phase in the interface and this model can deal with the unequal interfacial energies. It should be noticed that when $p_{k}=0$, this free energy functional reads

$$
F=\int\left(\frac{\varepsilon^{2}}{2}\left(\nabla p_{i}\right)^{2}+\omega_{i j} p_{i}^{2}\left(1-p_{i}\right)^{2}+\widetilde{g}\left(p_{i}\right) f_{i}^{0}\left(c_{i}, T\right)+\left(1-\widetilde{g}\left(p_{i}\right)\right) f_{j}^{0}\left(c_{j}, T\right)\right) \cdot d V
$$

This is exactly the same as the free energy functional proposed in the quantitative phase-field modeling for the single phase solidification [21].

\subsection{Time evolution equations}

As mentioned in the previous section, the time evolution of phase fields is described by the time-dependent Ginzburg-Landau equation (2). In the free energy functional given in 
eq.(5), three concentration fields, $\left\{c_{i}\right\}$, are used as the variables. As in the conventional multi-phase-field models [6-9], these concentrations obey the mixture rule, $c=\Sigma p_{i} c_{i}$. Also, following Kim, Kim, Suzuki's (KKS) model [23], the condition for the equal chemical potential, $\partial f_{i} / \partial c_{i}=\partial f_{j} / \partial c_{j}=\partial f_{k} / \partial c_{k}=\mu_{c}$, is introduced to make the steady state profiles of the phase fields independent of the concentration fields in the interface region. Then, the functional derivative of $F$ on the right-hand side of eq.(3) is written as,

$$
\begin{aligned}
\frac{\delta F}{\delta p_{i}}=-\frac{1}{2} \varepsilon^{2} \nabla^{2} p_{i} & +2 \omega\left(\left\{p_{i}\right\}\right) p_{i}\left(1-p_{i}\right)\left(1-2 p_{i}\right) \\
+ & \frac{\partial \omega\left(\left\{p_{i}\right\}\right)}{\partial p_{i}}\left(\sum_{j} p_{j}{ }^{2}\left(1-p_{j}\right)^{2}\right)+\sum_{j} \frac{\partial g_{j}}{\partial p_{i}}\left(f_{j}-\mu_{c} c_{j}\right) .
\end{aligned}
$$

The last term on the right-hand side of eq.(11) represents the thermodynamic driving force for the solidification. In dilute solution limit, the driving force term can be approximated as,

$$
f_{j}-\mu_{c} c_{j} \approx v_{m}^{-1} R T\left(-c_{k, e}^{j}+c_{j, e}^{k}-c_{j}\right),
$$

where $v_{m}$ is the molar volume, $R$ is the gas constant and $c_{j, e}^{k}$ is the concentration in $j$ phase in equilibrium with $k$ phase. In the expression of eq.(12), $k$ phase corresponds to a reference phase. When $j=k, f_{k}-\mu_{c} c_{k}$ is given as $-v_{m}{ }^{-1} R T c_{k}$. In our numerical computation, the reference phase was chosen to be the liquid phase.

The time evolution equation (2) is different from the one in the conventional model in the light of the fact that a different value is assigned to the mobility for different two-phase interface in the conventional model. In eq.(2), we introduced the dependence of the mobility on the phase field in order to handle the different mobility for the different interface without 
the formation of the extra phase [20]. $M\left(\left\{p_{j}\right\}\right)$ is defined in this study as follows,

$$
M\left(\left\{p_{j}\right\}\right)=2\left(\sum_{i, j>i} M_{i j} p_{i} p_{j}\right)\left(\sum_{i, j>i} p_{i} p_{j}\right)^{-1}
$$

where $M_{i j}$ is the mobility for $i-j$ interface. When $p_{k}=0, M\left(\left\{p_{i}\right\}\right)$ is given as $M\left(\left\{p_{i}\right\}\right)=2 M_{i j}$. This function contains the discontinuity at $p_{k}=1$. However, it does not cause any problem in practice.

The time evolution of the concentration field, $c$, is described by the following diffusion equation,

$$
\frac{\partial c}{\partial t}=-\nabla\left(\mathbf{J}_{c}+\mathbf{J}_{a t}\right)
$$

where $\mathbf{J}_{\mathrm{c}}$ is the solute flux and $\mathbf{J}_{a t}$ is the antitrapping current. $\mathbf{J}_{\mathbf{c}}$ is given as [6],

$$
\mathbf{J}_{c}=-D\left(\left\{p_{i}\right\}\right) \sum_{i} p_{i} \nabla c_{i}
$$

where $D\left(\left\{p_{i}\right\}\right)$ is the diffusion coefficient of $p_{i}$ dependent. In this study, we use the relation $c_{i}=k_{i j} c_{j}$ with the partition coefficient $k_{i j}=c_{i} / c_{j}$, since our focus is placed on the dilute solid solution. To keep the consistency with the single phase solidification model [21], in this study, we define $D\left(\left\{p_{i}\right\}\right)$ as follows,

$$
D\left(\left\{p_{i}\right\}\right)=\left(\sum_{i} D_{i} k_{i j} p_{i}\right)\left(\sum_{i} k_{i j} p_{i}\right)^{-1},
$$

with a reference phase $j . D_{i}$ is the diffusion coefficient of solute element in $i$ phase. Then, the solute flux is expressed by the relatively simple form,

$$
\mathbf{J}_{c}=-\sum_{i} D_{i} p_{i} \nabla c_{i} .
$$


An assumption should be necessarily introduced in the extension of the antitrapping current approach to the two-phase solidification process, since no thin-interface limit analysis is available for the mixture state of three phases. We tested several possibilities for this extension and found that the approach proposed in Ref. [20] results in the best convergence behavior. Then, in this study, the antitrapping current for an arbitrary value of the solid diffusivity [21] is extended as follows,

$$
\mathbf{J}_{a t}=-2 \sum_{i, j>i}\left(\mathbf{n}_{i} \cdot \mathbf{n}_{j}\right) a_{i j} \frac{\varepsilon}{\sqrt{\omega_{i j}}}\left(c_{j}-c_{i}\right) \frac{\partial p_{i}}{\partial t} \mathbf{n}_{i},
$$

where $\mathbf{n}_{i}$ denotes the unit vector normal to the contour surface of $p_{i}$ phase field as given by $\mathbf{n}_{i}=-\nabla p_{i} /\left|\nabla p_{i}\right| \cdot a_{i j}$ is the coefficient of which the form depends on the type of interpolating functions used in the model. In the present model, the coefficient $a_{i j}$ is given by [21],

$$
a_{i j}=\frac{1}{2 \sqrt{2}}\left[D_{j}-k_{i j} D_{i}\right] \Psi_{i j}\left(\chi_{i j}\right)
$$

with

$$
\Psi_{i j}\left(\chi_{i j}\right)=1-\frac{1}{2}\left[1-k_{i j} D_{i} / D_{j}\right] \chi_{i j}
$$

where $\chi_{i j}$ is a quantity associated with the solute concentration field at the $i-j$ interface. As discussed in Ref. [21], in this approach, $\chi_{i j}$ is regarded as a parameter controlling the convergence behavior of the output with respect to the interface thickness, $W$.

The most important fact in the present model is that the equations (2) and (14) reduce to the following equations at $p_{k}=0$, 


$$
\begin{aligned}
& \frac{1}{M_{i j}} \frac{\partial p_{i}}{\partial t}=\varepsilon^{2} \nabla^{2} p_{i}-2 \omega_{i j} p_{i}\left(1-p_{i}\right)\left(1-2 p_{i}\right)-30 p_{i}^{2}\left(1-p_{i}\right)^{2}\left(f_{i}-f_{j}-\left(c_{i}-c_{j}\right) \mu_{c}\right), \\
& \frac{\partial c}{\partial t}=\nabla\left[\left(D_{j}+\left(D_{i} k_{i j}-D_{j}\right) p_{i}\right) \nabla c_{j}+2 a_{i j} \frac{\varepsilon}{\sqrt{\omega_{i j}}}\left(c_{j}-c_{i}\right) \frac{\partial p_{i}}{\partial t} \frac{\nabla p_{i}}{\left|\nabla p_{i}\right|}\right] .
\end{aligned}
$$

These equations are exactly identical to those in the quantitative phase-field model developed in Ref. [21]. It should not be necessary to repeat here the asymptotic analysis of these equations. It was demonstrated in Ref. [21] that the model given by eqs. (21) and (22) can be mapped onto the FBP in the thin-interface limit and it allows the quantitatively accurate simulation with a finite value of $W$. Compared with the earlier work in Ref. [20], the present model is advantageous in terms of the fact that the solid diffusions and the unequal interfacial energies are taken into account.

\subsection{Relationships between parameters}

The relationships between the phenomenological parameters in the phase-field model and the measurable quantities are discussed. For a planar $i-j$ interface, the steady state profile of $p_{i}$ phase field is analytically expressed by solving eq.(21) as follows,

$$
p_{i}=\frac{1}{2}\left(1-\tanh \left(\frac{x}{\sqrt{2} W_{i j}}\right)\right) \text {, }
$$

where $W_{i j}=\varepsilon /\left(\omega_{i j}\right)^{1 / 2}$ corresponds to the thickness of $i-j$ interface. Also, the $i-j$ interfacial energy, $\sigma_{i j}$, is represented by

$$
\sigma_{i j}=\frac{1}{3 \sqrt{2}} \varepsilon \sqrt{\omega_{i j}}
$$


It is noted that $\varepsilon$ does not depend on the type of interface and, hence, $W_{i j}$ should be chosen so that the product $\sigma_{i j} W_{i j}$ is independent of the type of interface. Then, $\varepsilon$ and $\omega_{i j}$ are uniquely determined under a given set of $\sigma_{i j}$.

According to the thin-interface limit analysis, the mobility $M_{i j}$ is given, under the condition of vanishing kinetic coefficient, as follows [21],

$$
M_{i j}=\left(\frac{15}{4} \frac{a_{2} \cdot \varepsilon^{2}}{\omega_{i j} \cdot D_{j}} \frac{R T_{m, i j}}{v_{m}}\left(1-k_{i j}\right)\left(c_{j, e}^{i}-c_{i, e}^{j}\right) \Psi_{i j}\left(\chi_{i j}\right)\right)^{-1},
$$

where $T_{m, i j}$ is the transition temperature between $i$ and $j$ phases of pure element and $a_{2}$ is the constant depending on the forms of interpolating functions used in this model and it is given in the present model as $a_{2}=0.6276 \cdots$.

\section{Computational details}

We carried out the simulation for the peritectic reaction of carbon steel, $\operatorname{liquid}(\mathrm{L})+$ ferrite $(\delta) \rightarrow$ austenite $(\gamma)$, based on the present model to check the convergence behavior of the output with respect to the interface thickness, $W_{i j}$. For convenience, in the following discussion, we use the notation $\gamma, \delta$ and $\mathrm{L}$ for the index in variable such as $p_{\mathrm{L}}, \sigma_{\gamma \delta}$ instead of $i=1,2$ and 3 .

Equations (2) and (14) were discretized based on standard second-order finite difference formula with the squire grid spacing, $\Delta x$. We performed two-dimensional simulations for the isothermal peritectic reaction process, focusing on the growth of plate-like 
$\gamma$ phase. The system of our focus is schematically illustrated in Fig. 1. The system has a square shape with zero flux boundary condition along all the boundaries. The edge lengths are denoted by $W_{x}$ and $W_{y}$. There is a flat $\delta$ - $\mathrm{L}$ interface. The initial concentrations of $\delta$ and $\mathrm{L}$ phases are given as $c_{\delta, e}^{L}$ and $c_{L, e}^{\delta}$, respectively, at the holding temperature. In the initial state, there is a semicircular $\gamma$ phase with a diameter, $d_{i}$, and a concentration, $c_{\gamma}{ }^{0}$.

The $\gamma$ phase grows along the $\delta$-L interface ( $y$ direction). The velocity of the front edge of the growing $\gamma$ phase, viz., the peritectic reaction rate was calculated at several degrees of undercooling from the peritectic reaction temperature, $\Delta T$, until the steady state was realized. Our preliminary simulations showed that the velocity takes almost a constant value typically after $t=2.0 \times 10^{-4} \mathrm{~s}$ for $\Delta T=5 \mathrm{~K}$ and $t=1.0 \times 10^{-4} \mathrm{~S}$ for $\Delta T=10 \mathrm{~K}$. The time step was set to be $\Delta t=5 \times 10^{-11}-2 \times 10^{-10} \mathrm{~s}$, depending on $\Delta x$ and $\Delta T$. It was observed in our preliminary simulations that small system size incurs a size effect that the steady state velocity appreciably depends on the values of $W_{x}$ and $W_{y}$. The values of $W_{x}$ and $W_{y}$ were accordingly chosen in each condition to be large enough to avoid such a size effect. On the other hand, the values of $c_{\gamma}{ }^{0}$ and $d_{i}$ were found to be irrelevant to the steady state velocity. In all the calculations presented here, we employed $c_{\gamma}^{0}=c_{\gamma, e}^{L}$ and $d_{i}=40 \times 10^{-8} \mathrm{~m}$. The interface thicknesses were set to be $W_{\gamma \delta}=2.0 \times \Delta x, W_{\delta \mathrm{L}}=\sigma_{\gamma \delta} W_{\gamma \delta} / \sigma_{\delta \mathrm{L}}$ and $W_{\gamma \mathrm{L}}=\sigma_{\gamma \delta} W_{\gamma \delta} / \sigma_{\gamma \mathrm{L}} . \omega_{t r i}$ in eq.(8) was given as $\omega_{t r i}=\omega_{\delta \mathrm{L}}+\omega_{\gamma \mathrm{L}}+\omega_{\gamma \delta}$, unless otherwise stated. The parameter $\chi_{i j}$ was set to be $\chi_{i j}=0$ for all the interfaces. The computation with $\chi_{i j}=0$ yields quite reasonable convergence 
behavior for the dendrite growth [21] and this is also the case for the peritectic reaction, as demonstrated in the next section. The other input parameters employed in this study are listed in Table 1.

\section{Results and discussion}

As described in the introduction, the conventional models involve the anomalous interface effects and the magnitude of these effects scales with the interface thickness. Shown in Fig. 2 is the dependence of the growing velocity of $\gamma$ phase (peritectic reaction rate), $V_{p}$, on the interface thickness $W_{\gamma \delta}$. The degree of undercooling is $\Delta T=10 \mathrm{~K}$. In these calculations, the thicknesses of the $\delta$-L and $\gamma$-L interfaces were also varied according to the relations $W_{\delta \mathrm{L}}=\sigma_{\gamma \delta} W_{\gamma \delta} / \sigma_{\delta \mathrm{L}}$ and $W_{\gamma \mathrm{L}}=\sigma_{\gamma \delta} W_{\gamma \delta} / \sigma_{\gamma \mathrm{L}}$ as discussed in the previous section. The values of $\varepsilon, \omega_{i j}$, $\omega_{t r i}$ and $M_{i j}$ were varied accordingly. The system size was chosen to be $W_{x} \geq 4 \times 10^{-6} \mathrm{~m}$ and $W_{y} \geq$ $2 \times 10^{-6} \mathrm{~m}$. Three types of square symbols represent the results calculated with the assumption of the equal interfacial energies, $\sigma_{\delta \mathrm{L}}=\sigma_{\gamma \mathrm{L}}=\sigma_{\gamma \delta}=2.04 \mathrm{~J} \cdot \mathrm{m}^{-2}$. The full square and half-open square symbols indicate the results for $n=1.4$ and $n=2.0$, respectively. The reason for the choice of the special value, $n=1.4$, will be explained later. The open square symbols represent the results for $n=0.0$ and $\omega_{\text {tri }}=0.0$. In this case, the model is actually equivalent to the model of Ref. [20], except that the solid diffusions are taken into account in the present model. One can see that all the results merge into almost the single curve as the interface thickness decreases. 
However, a well converged value seems to be not achieved even for the smallest value of $W_{\gamma \delta}$ tested here. Importantly the convergence behavior depends on the value of $n$. The simulation with $n=1.4$ yields the most reasonable convergence behavior. It is noticed that the extra phase does not form in each interface region in the simulation for the equal interfacial energies. The difference in the convergence behavior originates from the difference in the potential height for the mixture state of three phases which is called the additional potential here. Among these cases, the additional potential height is the lowest in the simulation for $n=0.0$ and is the highest for $n=1.4$. Hence, it is seen that the increase in the additional potential height leads to the better convergence behavior, which should be ascribable to narrowing of the region for the mixture state of three phases near the triple junction.

Three types of the circular symbols in Fig. 2 indicate the velocities calculated with the unequal interfacial energies listed in Table 1. The results for $n=1.4$ and $n=2.0$ are shown by full and half-full circle symbols, respectively. Also, the results for $n=0.0$ and $\omega_{\text {tri }}=0.0$ are indicated by the open symbols. The simulation with the unequal interfacial energies involves the problem associated with the extra phase in the interface. Especially, the extra phase is prone to form in the interface of the highest interfacial energy, specifically, the $\gamma-\delta$ interface in this system. The phase field profiles across the $\gamma-\delta$ interface far from the triple junction are shown in Fig. 3(a). The result for $n=1.4$ and $W_{\gamma \delta}=1.0 \times 10^{-8} \mathrm{~m}$ is presented in Fig. 3(a). The enlargement of the phase field profiles in the vicinity of $p_{i}=0$ is shown in Fig. 3(b) where the 
profiles of $p_{\mathrm{L}}$ for $n=0.0$ and 2.0 are added. It is seen that the spatial profile of $p_{\mathrm{L}}$ depends on the value of $n$. When there is no additional potential $\left(n=0.0\right.$ and $\left.\omega_{t r i}=0.0\right)$, the extra phase exists in the $\gamma-\delta$ interface. The peak position of $p_{\mathrm{L}}$ is located not at the center of the $\gamma-\delta$ interface but on the $\delta$ phase side. In other words, the $\delta$-L interface configuration appears inside the $\gamma-\delta$ interface. This is because the $\delta$-L interface possesses the lowest interfacial energy in this system and it incurs the tendency of the $\delta$-L interface configuration to form. The maximum value of $p_{\mathrm{L}}$ is reduced by introducing the additional potential $(n=2.0)$. Most importantly, the extra phase vanishes for $n=1.4$. It is clear in Fig. 2 that the simulation for $n=1.4$ exhibits the excellent convergence behavior.

In Fig. 3(b), the peak value of $p_{\mathrm{L}}$ for $n=0.0$ is higher than the one for $n=2.0$. However the difference in the peak value is not significantly large. Therefore, there is actually only a slight difference between these cases in the migration velocity of the $\gamma-\delta$ interface far from the triple junction. The appreciable difference between the peritectic reaction rates in Fig. 2 (especially at $W_{\gamma \delta}=1.0 \times 10^{-8} \mathrm{~m}$ ) originates from the formation of the extra phase near the triple junction. For this discussion, we employ the square sum of phase fields, $p_{s}=p_{\mathrm{L}}{ }^{2}+p_{\delta}{ }^{2}+p_{\gamma}{ }^{2} \cdot p_{s}$ varies from 0.5 to 1.0 across the two-phase interface and it takes the minimum value of $p_{s}=1 / 3$ at the triple junction. The contour lines of $p_{s}$ near the triple junction for $n=0.0,1.4$ and 2.0 are shown in Fig. 4. These are the results calculated with $W_{\gamma \delta}=1.0 \times 10^{-8} \mathrm{~m}$. The solid lines indicate the level 0.5 contour lines of phase fields. The cross-hatched region represents the region for 
$p_{s} \leq 0.5$ and, hence, the mixture state of three phases forms in this region. It is clear that the mixture state is confined into a narrow region when the additional potential contribution is large (Fig. 4(b)). When the contribution of the additional potential is reduced (Fig. 4(c)), the region for the mixture state is extended to each interface region and especially to the $\gamma-\delta$ interface region. When the addition potential is removed (Fig. 4(a)), the region for the mixture state is substantially extended to the $\gamma-\delta$ interface region. Then, the motion of the interface in this region cannot be appropriately described due to the existence of the extra phase. The antitrapping current does not properly work and the anomalous interface effects become significant in this region. As a result, the peritectic reaction rate calculated for $n=0.0$ is quite different from those for $n=2.0$ and 1.4 even for the smallest value of $W_{\gamma \delta}$ in Fig. 2. In addition, as detailed in the supplementary material, the formation of the extra phase cannot be suppressed only by decreasing the interface thickness when the additional potential is not introduced. In Fig. 2, therefore, the result for $n=0.0$ seems to be converged to a different value from the one for $n=1.4$.

In the present model, the contribution of the additional potential increases with the decrease in $n$ and with the increase in $\omega_{\text {tri }}$. As discussed in the supplementary material, the formation of the extra phase can be effectively suppressed by using the small value of $n$ and/or the larger value of $\omega_{t r i}$ in the present model. The convergence behavior can be thereby improved. However, when the contribution of the additional potential is too large, the 
calculation becomes unstable. When we tried $n=1.2$ and 1.3 with $\omega_{t r i}=\omega_{\delta \mathrm{L}}+\omega_{\gamma \mathrm{L}}+\omega_{\gamma \delta}$, the calculations were not stable. Since the excellent convergence was demonstrated for $n=1.4$ and $\omega_{t r i}=\omega_{\mathrm{L} \delta}+\omega_{\mathrm{L} \gamma}+\omega_{\delta \gamma}$, we employed these values in the following calculations.

The dependence of $V_{\mathrm{p}}$ at $\Delta T=10 \mathrm{~K}$ on $W_{\gamma \delta}$ is shown in Fig. 5(a). These are the results for the unequal interfacial energies. For comparison, we performed the same simulation based on a model without the antitrapping current and with $D\left(\left\{p_{i}\right\}\right)=D_{L} p_{L}+D_{\delta} p_{\delta}+D_{\gamma} p_{\gamma}$ in eq.(15), which is called the standard model here. It is noted that this standard model is free from the problem of the extra phase in the interface, while it involves the spurious interface effects in the motion of the two-phase interface (chemical potential jump at the interface and surface diffusion). It is seen that the present model exhibits the well-converged value even for a relatively large value of $W_{\gamma \delta}=3.0 \times 10^{-8} \mathrm{~m}$. On the other hand, the result of the standard model largely depends on the interface thickness and the result is not well converged even for the smallest value of $W_{\gamma \delta}=1.0 \times 10^{-8} \mathrm{~m}$ tested here. The same simulations were performed for $\Delta T=5 \mathrm{~K}$ and the results are shown in Fig. $5(\mathrm{~b})$. The system size was chosen to be $W_{x} \geq 6 \times 10^{-6} \mathrm{~m}$ and $W_{y} \geq 3 \times 10^{-6} \mathrm{~m}$. The smallest value of $W_{\gamma \delta}$ was set to be $2.0 \times 10^{-8} \mathrm{~m}$ in the light of the computational burden. It is again demonstrated that the convergence behavior obtained by the present model is excellent as compared with that by the standard model.

The steady state shape of the interfaces near the triple junction at $\Delta T=10 \mathrm{~K}$ is shown in Fig. 6 (a). The origin of $y$ axis corresponds to the center of triple junction and the origin of 
$x$ axis is the initial position of $\delta$ - $\mathrm{L}$ interface. These lines represent the level 0.5 contour lines of phase fields. The shape of the interfaces near the triple junction is almost independent of the interface thickness in the present model, while the results of the standard model depend on the interface thickness. In Fig. 6(a), the deviation of the $\delta$-L interface from the initial position indicates the melting of $\delta$ phase near the growth front of $\gamma$ phase. This steady state shape near the junction represents the well-known feature in the peritectic reaction process, as recently demonstrated in the quantitative phase-field simulation without the solid diffusion [29]. More detailed analysis on the peritectic reaction in the carbon steel is reported elsewhere [30]. Figure 6 (b) shows the concentration profiles along $x$ direction at $y=-0.3 \mu \mathrm{m}$. The circular and square symbols represent the results of the present and the standard models, respectively. One can see that the concentration profiles are also independent of the interface thickness in the present model.

As demonstrated above, the present model exhibits the excellent convergence behavior of the outcomes with respect to the interface thickness. Although the detail is not discussed here, it was confirmed that the present model successfully reproduces the equilibrium relation of contact angles between interfaces near the triple junction described by Young's rule within an accuracy of less than $1^{\circ}$ near the peritectic temperature [30].

\section{Conclusions}


In this study, we developed the quantitative phase-field model for two-phase

solidification processes. This model is consistent with the available quantitative phase-field model for the single phase solidification. Hence, the free boundary problem is recovered with a finite value of interface thickness in describing the motion of the interfaces. Importantly, this model can be applied to the system with arbitrary values of the solid diffusivities and interfacial energies. In this study, we investigated the performance of this model, focusing on the peritectic reaction in the carbon steel. The excellent convergence behavior of the outcome was demonstrated. The extension of the present approach to the multi-component system is quite important task for a step further to the development of effective computational tool to quantitatively describe the microstructural evolution processes.

\section{Acknowledgments}

This work is supported by $18^{\text {th }}$ ISIJ Research Promotion Grant and Grant-in-Aid for Challenging Exploratory Research 21656192 from MEXT, Japan. Also, the author M.O. acknowledges partial financial support from the Next Generation Super Computing Project, Nano-science Program, MEXT, Japan. 


\section{References}

[1] A. Karma, Encyclopedia of Materials Science and Technology (Elsevier, Oxford, 2001).

[2] L.-Q. Chen, Annu Rev Mater Sci 2002;32;113.

[3] I. Steinbach, Modelling Simul Mater Sci Eng 2009;17; 073001.

[4] I. Steinbach, F. Pezzola, B. Nestler, M. Seeßelberg, R. Prieler, G.J. Schmitz and J.L.L. Rezende, Physica D 1996;94;135.

[5] I. Steinbach and F. Pezzola, Physica D 1999;134;385.

[6] S.G. Kim, W.T. Kim, T. Suzuki, M. Ode, J Cryst Growth 2004;261;135.

[7] B. Boettger, J. Eiken and I. Steinbach, Acta Mater 2006;54;2697.

[8] R.S. Qin, E.R. Wallach and R.C. Thomson, J Cryst Growth 2005;279;163.

[9] J. Eiken, B. Böttger and I. Steinbach, Phys Rev E 2006;73;0666122.

[10] R.F. Almgren, SIAM J Appl Math 1999;59;2086.

[11] A. Karma and W.-J. Rappel, Phys Rev E 1998;57;4323.

[12] A. Karma, Phys Rev Lett 2001;87;115701.

[13] B. Echebarria, R. Floch, A. Karma and M. Plapp, Phys Rev E 2004;70;061604.

[14] J.C. Ramirez, C. Beckermann, A. Karma and H.-J. Diepers, Phys Rev E 2004;69;051607.

[15] M. Greenwood, M. Haataja and N. Provatas, Phys Rev Lett 2004;69;051607.

[16] C.W. Lan and C.J. Shih, Phys Rev E 2004;69;031601.

[17] J.C. Ramirez and C. Beckermann, Acta Mater 2005;53;1721.

[18] A. Badillo and C. Beckermann, Acta Mater 2006; 54; 2015.

[19] S.G. Kim, Acta Mater 2007;55;4391.

[20] R. Folch and M. Plapp, Phys Rev E 2005; 72;011602.

[21] M. Ohno and K. Matsuura, Phys Rev E 2009;79;031603.

[22] H. Gracke, B. Nestler and B. Stoth, SIAM J Appl Math, 1999;60;295.

[23] S.G. Kim, W.T. Kim and T. Suzuki, Phys Rev E 1999;60;7186.

[24] W. Kurz and D.J. Fisher, Fundamentals of Solidification, Trans Tech Publication (Switzerland, 1989).

[25] Y. Sun, M. Asta, J.J. Hoyt, M.I. Mendelev and D.J. Srolovitz, Phys Rev B 2004;69;020102(R).

[26] H. Yin, T. Emi and H. Shibata, Acta Mater 1999;47;1523.

[27] T. Matsumiya, H. Kajioka, S. Mizoguchi, Y. Ueshima and H. Esaka, Trans ISIJ 1984;24;873.

[28] P. Gustafson, Scand J Metall 1985;14;259.

[29] G. Boussinot, E.A. Brener, D.E. Temkin, Acta Mater 2010;58;1750.

[30] M. Ohno and K. Matsuura, (submitted). 
Table 1 Input parameters for Fe-C system used in the present study

\begin{tabular}{|c|c|c|}
\hline Quantity & Symbol & Value used \\
\hline Molar volume & $v_{m}$ & $7.7 \times 10^{-6} \mathrm{~m}^{3} / \mathrm{mol}[24]$ \\
\hline Partition coefficient of $\delta$-L phases & $k_{\delta \mathrm{L}}$ & $\left.0.179^{*}\right)$ \\
\hline Partition coefficient of $\gamma-\mathrm{L}$ phases & $k_{\gamma \mathrm{L}}$ & $\left.0.334^{*}\right)$ \\
\hline Partition coefficient of $\gamma-\delta$ phases & $k_{\gamma \delta}$ & $k_{\gamma \mathrm{L}} / k_{\delta \mathrm{L}}$ \\
\hline Melting temperature of pure bcc-Fe & $T_{m, \delta \mathrm{L}}$ & $1811 \mathrm{~K}^{*}$ \\
\hline Melting temperature of pure fcc-Fe & $T_{m, \gamma \mathrm{L}}$ & $1801 \mathrm{~K}^{*}$ \\
\hline$\delta / \gamma$ transition temperature of pure Fe & $T_{m, \gamma \delta}$ & $1399 \mathrm{~K}^{*}$ \\
\hline Liquidus slope of $\delta$ phase & $m_{\delta}$ & $1828 \mathrm{~K} /$ mol fraction $\left.^{*}\right)$ \\
\hline Liquidus slope of $\gamma$ phase & $m_{\gamma}$ & $1399 \mathrm{~K} / \mathrm{mol}$ fraction $^{*}$ \\
\hline Interfacial energy of $\delta$-L boundary & $\sigma_{\delta \mathrm{L}}$ & $0.204 \mathrm{~J} / \mathrm{m}^{2}[24]$ \\
\hline Interfacial energy of $\gamma$-L boundary & $\sigma_{\gamma \mathrm{L}}$ & $0.319 \mathrm{~J} / \mathrm{m}^{2}[25]$ \\
\hline Interfacial energy of $\gamma-\delta$ boundary & $\sigma_{\delta \gamma}$ & $0.370 \mathrm{~J} / \mathrm{m}^{2}[26]$ \\
\hline Diffusion coefficient in L phase & $D_{\mathrm{L}}$ & $\left.5.2 \times 10^{-7} \cdot \exp \left(-5.0 \times 10^{4} \cdot \beta\right) \mathrm{m}^{2} / \mathrm{s}^{* *}\right)[27]$ \\
\hline Diffusion coefficient in $\delta$ phase & $D_{\delta}$ & $\left.1.27 \times 10^{-6} \cdot \exp \left(-8.3 \times 10^{4} \cdot \beta\right) \mathrm{m}^{2} / \mathrm{s}^{* *}\right)[27]$ \\
\hline Diffusion coefficient in $\gamma$ phase & $D_{\gamma}$ & $\left.7.61 \times 10^{-6} \cdot \exp \left(-13.7 \times 10^{4} \cdot \beta\right) \mathrm{m}^{2} / \mathrm{s}^{* *}\right)[27]$ \\
\hline
\end{tabular}




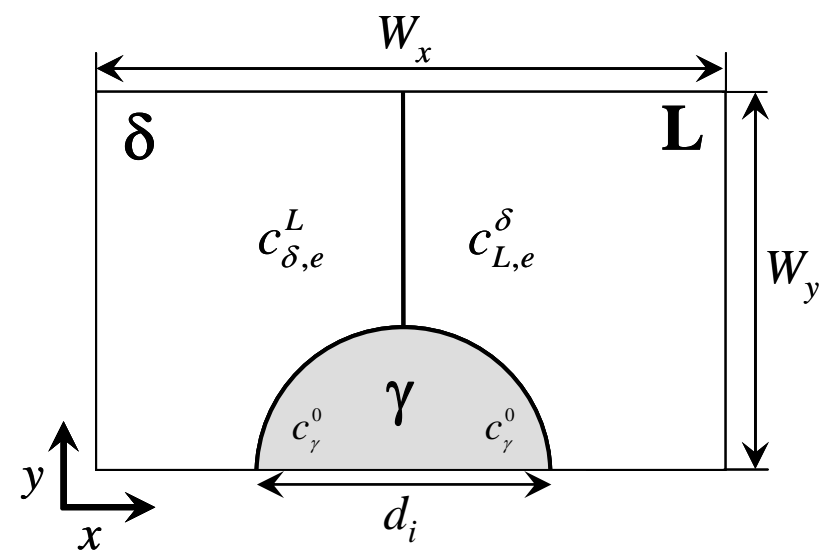

Fig. 1. Schematic illustration of the initial state in two-dimensional system used in this study. 


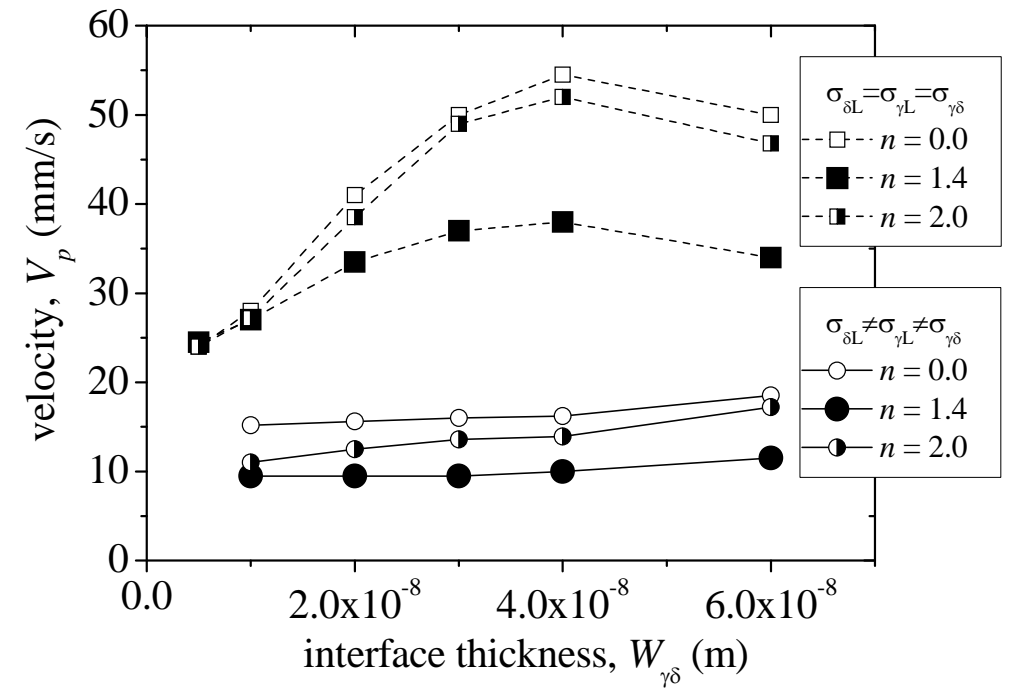

Fig. 2. Dependence of the growing velocity of $\gamma$ phase on interface thickness, $W_{\gamma \delta}$, calculated for $n=0.0$ (open symbols), $n=1.4$ (full symbols) and $n=2.0$ (half-full symbols). The degree of undercooling is $\Delta T=10 \mathrm{~K}$. The square symbols correspond to the results for equal interfacial energies, while the circular symbols represent those for unequal interfacial energies. 
(a)

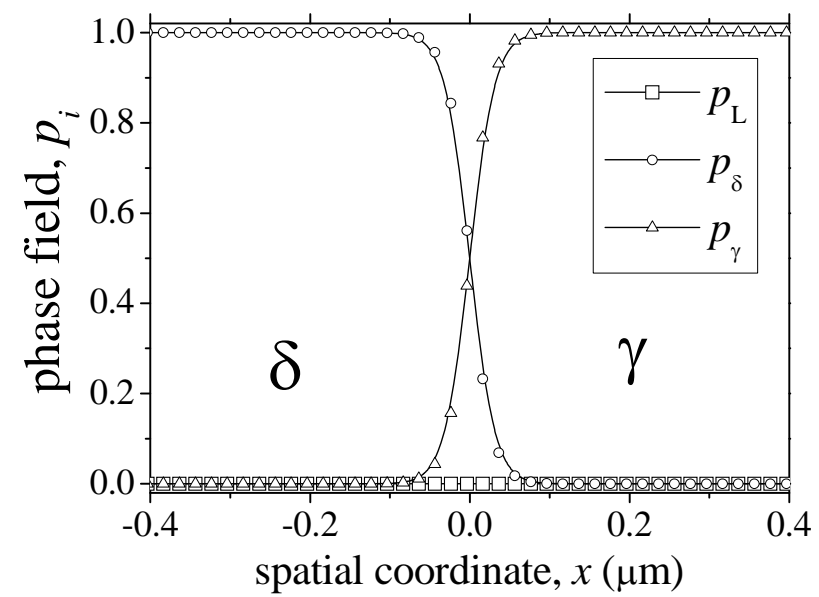

(b)

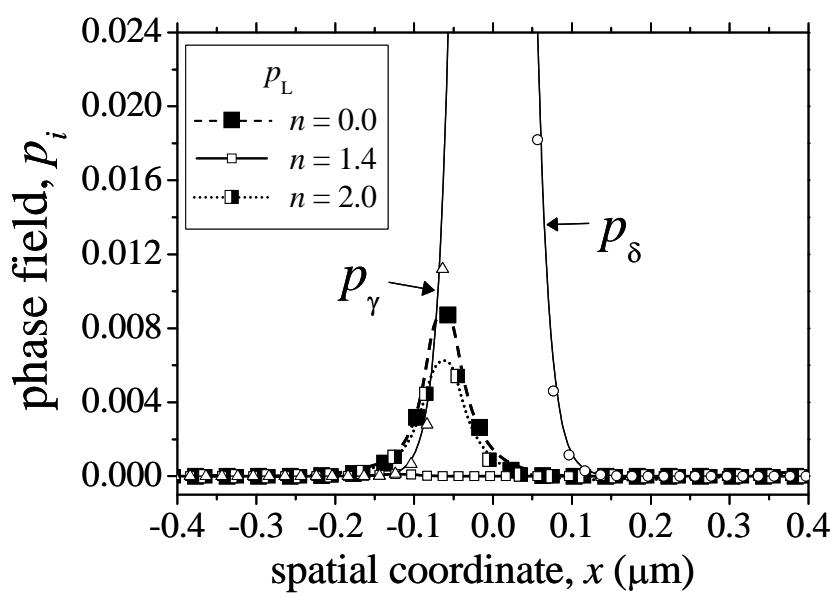

Fig. 3 (a) Phase field profiles across $\gamma-\delta$ interface along $x$ direction calculated for $n=1.4$ and $W_{\gamma \delta}=1.0 \times 10^{-8}$ $\mathrm{m}$ at $\Delta T=10 \mathrm{~K}$. For visual aid, one out of every 4 symbols is shown for each profile. (b) Enlargement of profile in the vicinity of $p_{i}=0$ where $p_{\mathrm{L}}$ profiles for $n=0.0$ and $n=2.0$ are also shown. One of every 8 symbols is shown for these profiles. 
(a)

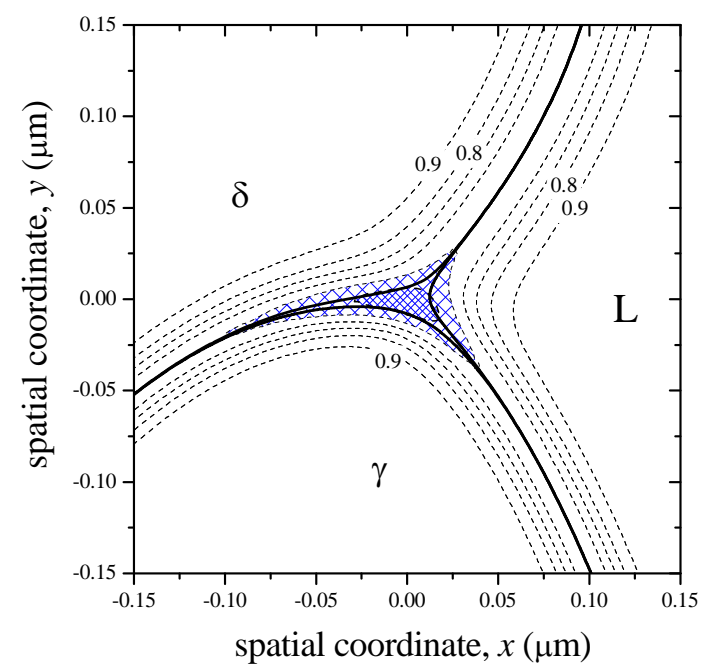

(c)

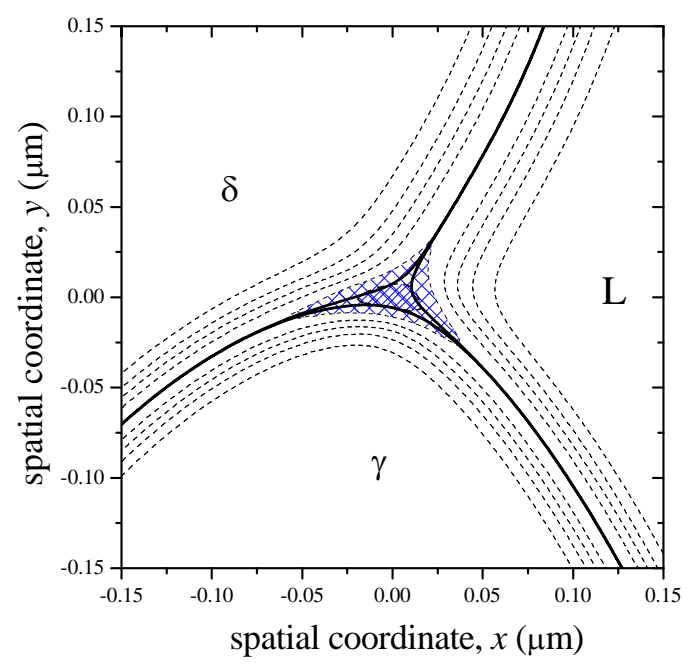

(b)

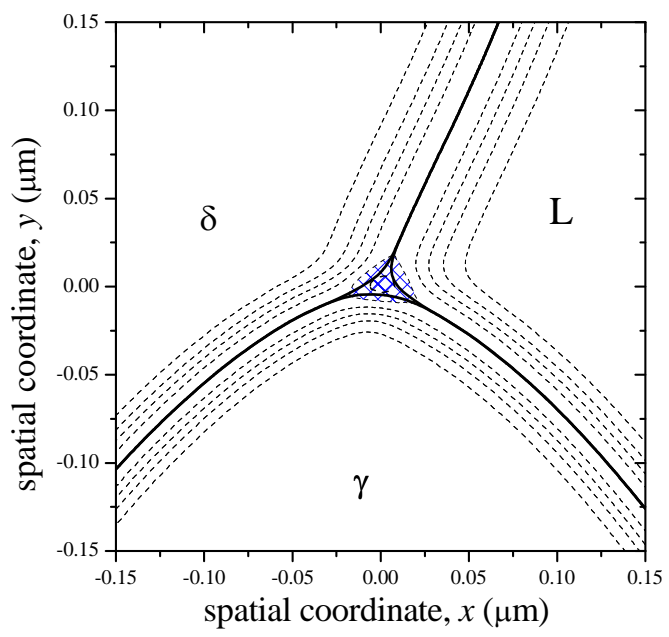

Fig. 4. Contour lines (dashed lines) for the square sum of phase fields, $p_{s}$, near the triple junction during the peritectic reaction, calculated (a) without the additional potential, (b) with $n=1.4$ and (c) with $n=2.0$. In each figures, the origin of the spatial coordinates correspond to the position of the triple junction $\left(p_{\mathrm{L}}=p_{\delta}=p_{\gamma}=1 / 3\right)$. The interval of the contour lines is $\Delta p_{\mathrm{s}}=0.1$. The cross-hatched region indicates the region where $p_{s} \leq 0.5$. The solid lines represent the level 0.5 contour lines of the phase fields. 


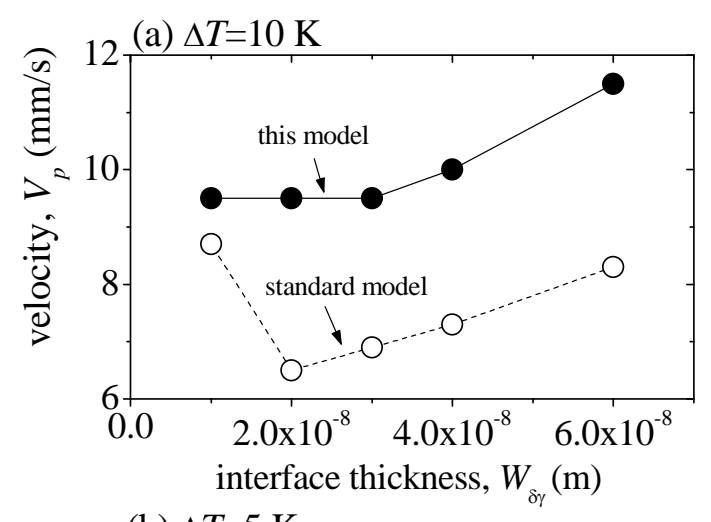

(b) $\Delta T=5 \mathrm{~K}$

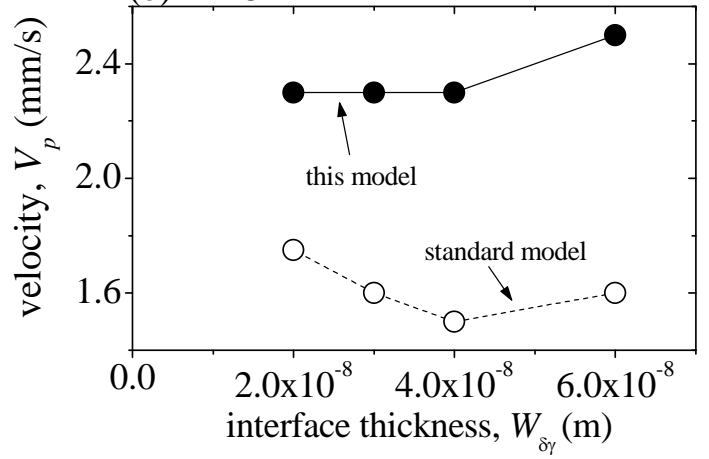

Fig. 5. Convergence behavior of the velocity of growing $\gamma$ phase with respect to the interface thickness, $W_{\gamma \delta}$, calculated for (a) $\Delta T=10 \mathrm{~K}$ and (b) $\Delta T=5 \mathrm{~K}$. The full and open symbols represent the results obtained by the present model and the standard model, respectively. 
(a)

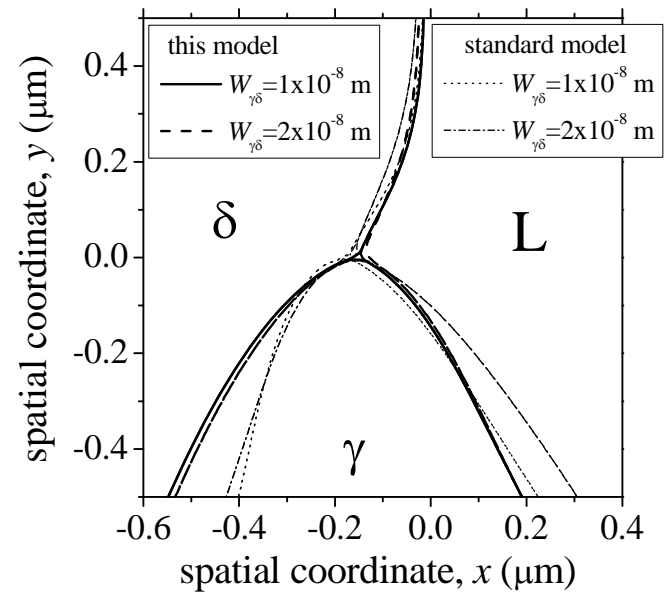

(b)

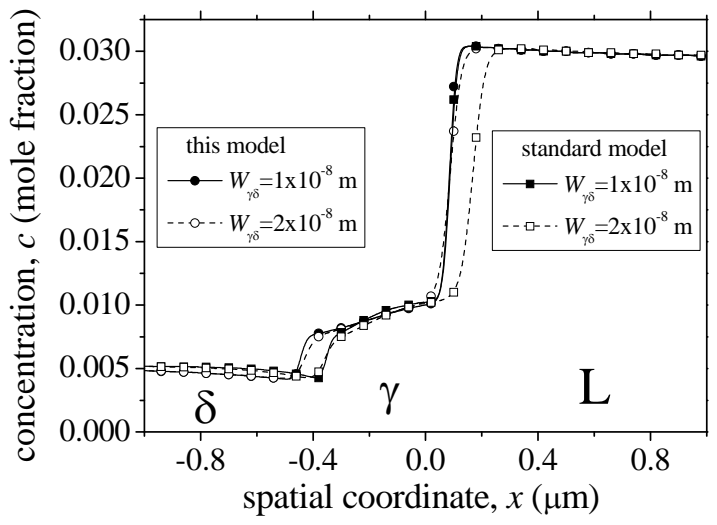

Fig. 6. (a) Level 0.5 contours of phase fields in the steady state at $\Delta T=10 \mathrm{~K}$. (b) Concentration profiles along $x$ direction at $y=-0.3 \mu \mathrm{m}$ in Fig. (a). For visual aid, one out of every 8 symbols is shown for each profile. 


\title{
Supplementary Material
}

\author{
A. Dependence of $\boldsymbol{f}_{\text {pot }}$ on $\boldsymbol{n}$ and $w_{t r i}$ \\ B. Difference in convergence behavior between the cases of equal and unequal \\ interfacial energies
}

Munekazu Ohno and Kiyotaka Matsuura

Division of Materials Science and Engineering, Faculty of Engineering, Hokkaido University, Sapporo, Hokkaido, 060-8628, Japan

May 24, 2010 


\section{A. Dependence of $\boldsymbol{f}_{\text {pot }}$ on $\boldsymbol{n}$ and $\boldsymbol{w}_{\text {tri }}$}

The Ginzburg-Landau-type free energy functional in the present model is described in eq.(5). The potential barrier between the bulk phases, $f_{\text {pot }}$, is written as follows,

$$
f_{p o t}=\left(2 \sum_{i, j>i} p_{i}{ }^{2} p_{j}{ }^{2}\right)^{-1}\left(\sum_{i, j>i} \omega_{i j} \cdot p_{i}{ }^{2} p_{j}{ }^{2}+\omega_{t r i} \cdot\left(p_{i} p_{j} p_{k}\right)^{n}\right)\left[\sum_{i} p_{i}{ }^{2}\left(1-p_{i}\right)^{2}\right],
$$

where $\omega_{i j}$ denotes the two-phase potential height between $i$ and $j$ phases. In eq.(S-1), the term, $\omega_{t r i} \cdot\left(p_{i} p_{j} p_{k}\right)^{n}$, represents the additional potential. The contribution of the additional potential to $f_{\text {pot }}$ is entirely determined by the values of $n$ and $\omega_{\text {tri }}$. Here, we show the dependence of $f_{\text {pot }}$ on the values on $n$ and $\omega_{\text {tri }}$.

As discussed in the section 2.3, $\omega_{i j}$ can be calculated as follows,

$$
\omega_{i j}=\frac{3 \sqrt{2} \cdot \sigma_{i j}}{W_{i j}}
$$

where $\sigma_{i j}$ and $W_{i j}$ are the interfacial energy and the interface thickness for $i-j$ interface, respectively. Here, we used the values of $\sigma_{i j}$ listed in Table 1 . The interface thicknesses were set to be $W_{\gamma \delta}=1 \times 10^{-8} \mathrm{~m}$ and $W_{\delta \mathrm{L}}=\sigma_{\gamma \delta} W_{\gamma \delta} / \sigma_{\delta \mathrm{L}}$ and $W_{\gamma \mathrm{L}}=\sigma_{\gamma \delta} W_{\gamma \delta} / \sigma_{\gamma \mathrm{L}}$. Figure S1 shows the contour lines of $f_{\text {pot }}$ calculated without the additional potential. The contour lines were drawn in the Gibbs simplex. The Gibbs simplex is the equilateral triangle, where each vertex represents the different phase, $\delta, \gamma$ or liquid. The value of the phase field is determined by the distance to the edge opposite to the corresponding vertex [20]. The interval of the contour lines is $\Delta f_{\text {pot }}=5 \times 10^{5} \mathrm{~J} / \mathrm{m}^{3}$. The local minima exist at each vertex. The two-phase potential height (the maximum value on the edge) is the highest at the $\gamma-\delta$ edge and is the lowest at the $\delta$-L edge. The global maximum exists not at the $\gamma-\delta$ edge but inside the simplex, a point very close to the $\gamma-\delta$ edge, although it cannot be clearly seen in Fig. S1. It is noted that the maximum value is not significantly higher than the two-phase potential height for the $\gamma-\delta$ interface. As described in the section 4, the $p_{\mathrm{L}}$ profile exhibits the "spike" in the $\gamma$ - $\delta$ interface when the additional potential is not introduced.

For convenience, we introduce the parameter, $\omega^{*}=\omega_{\delta \mathrm{L}}+\omega_{\gamma \mathrm{L}}+\omega_{\gamma \delta}$. Figure S2 shows the potential surfaces of $f_{\text {pot }}$ calculated for $\omega_{\text {tri }}=\omega^{*}$ and $n=1.1,1.4,2.0$ and 3.0. The potential surface for $n=3.0$ (Fig. S2(d)) is not substantially different from the one without the additional potential shown in Fig. S1. As the value of $n$ decreases, the maximum value increases and the maximum point gradually moves toward the center, namely, $p_{\mathrm{L}}=p_{\delta}=p_{\gamma}=1 / 3$. Figure S3 represents the potential surfaces calculated for $n=2.0$ 
and $\omega_{\text {tri }}=2 \omega^{*}, 3 \omega^{*}$ and $7 \omega^{*}$. As the value of $\omega_{\text {tri }}$ increases, the maximum value increases and the maximum point gradually moves toward the center.

From Figs. S1-S3, one can grasp that the decrease in $n$ and/or the increase in $\omega_{\text {tri }}$ raises the potential height for the mixture state of three phases. Therefore, the formation of the extra phase can be suppressed by using the small value of $n$ and the large value of $\omega_{t r i}$ in the present model. As shown in Fig. 2, we can improve the convergence behavior of the peritectic reaction rate with respect to the interface thickness by increasing the contribution of the additional potential. Therefore, we generally expect that the combination of the small value of $n$ and the large value of $\omega_{\text {tri }}$ yields the excellent result.

As shown in Figs. S2 and S3, the variation of the potential surface $f_{\text {pot }}$ due to the increment of $\omega_{\text {tri }}$ is similar to that due to the decrement of $n$. Hence, in our preliminary calculations, the value of $\omega_{\text {tri }}$ was fixed to be $\omega_{\text {tri }}=\omega^{*}$ and different values of $n$ were tested. When we tried $n=1.2$ and 1.3 with $\omega_{\text {tri }}=\omega^{*}$, the calculations were not stable in the present condition. Since the calculation for $n=1.4$ and $\omega_{\text {tri }}=\omega^{*}$ yields the reasonable convergence behavior without the formation of the extra phase, we employed $n=1.4$ and $\omega_{t r i}=\omega^{*}$ in the present study.

It is noted that the formation of the extra phase originates from the difference in $\sigma_{i j}$ and thus the difference in $\omega_{i j}$. From eq.(S-2), one can comprehend that the difference in $\omega_{i j}$ increases with the decrease in $W_{i j}$. Figure $\mathrm{S} 4$ shows the spatial profiles of $p_{\mathrm{L}}$ across the $\gamma-\delta$ interface far from the triple junction, calculated without the additional potential $(n=0.0)$ for $\Delta T=10 \mathrm{~K}$. It is seen that as $W_{\gamma \delta}$ decreases, the peak profile becomes sharp and the peak position moves toward the center position of the $\gamma-\delta$ interface. Importantly, the peak value increases with the decrease in $W_{\gamma \delta}$. Hence, the problem with the formation of the extra phase cannot be removed only by the reduction of the interface thickness. 


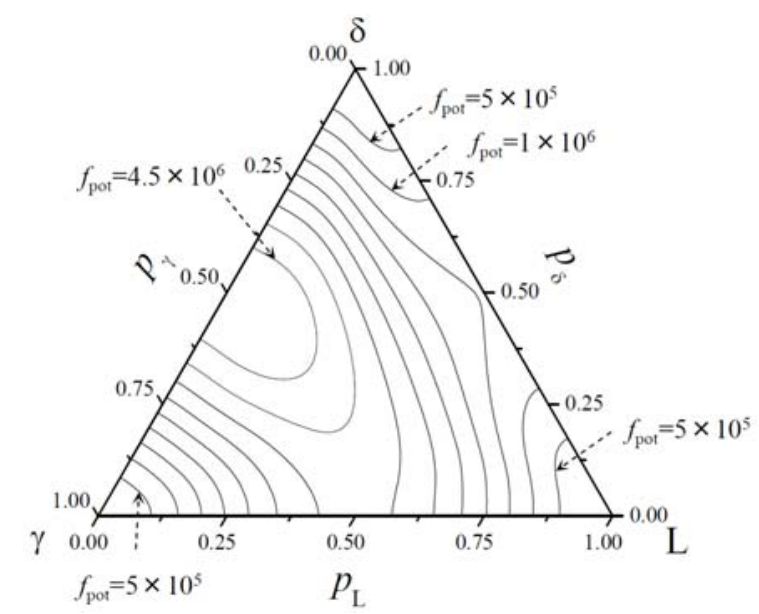

Fig. S1. Potential surface of $f_{\text {pot }}$ in the Gibbs simplex. The interval of the contour lines is $\Delta f_{\text {pot }}=5 \times 10^{5} \mathrm{~J} / \mathrm{m}^{3}$. The additional potential is not introduced.
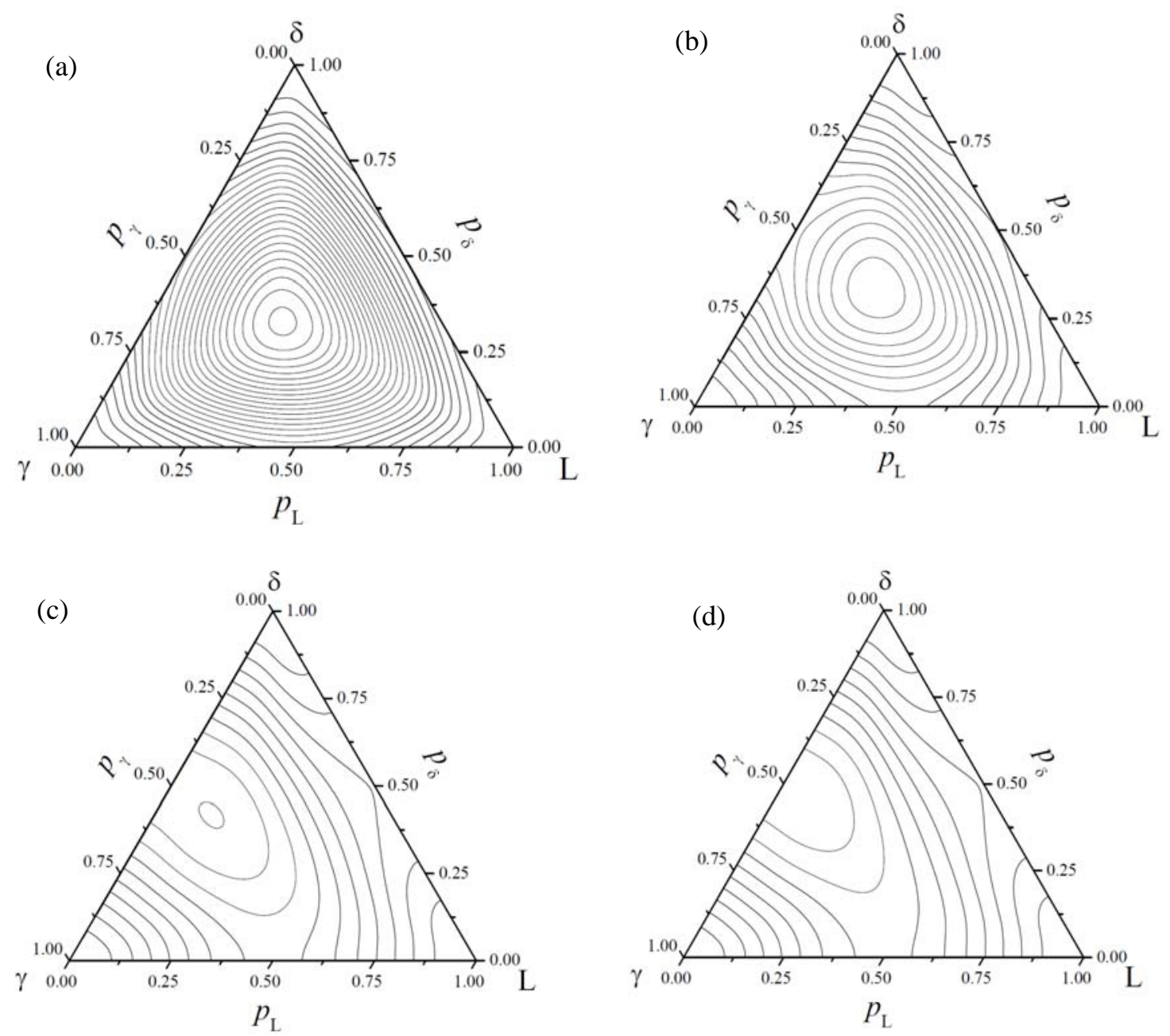

Fig. S2. Potential surfaces of $f_{\text {pot }}$ in the Gibbs simplex calculated for $\omega_{\text {tri }}=\omega^{*}$ and (a) $n=1.1$, (b) $n=1.4$, (c) $n=2.0$ and (d) $n=3.0$. In each figure, the interval of the contour lines is $\Delta f_{\text {pot }}=5 \times 10^{5} \mathrm{~J} / \mathrm{m}^{3}$. 
(a)

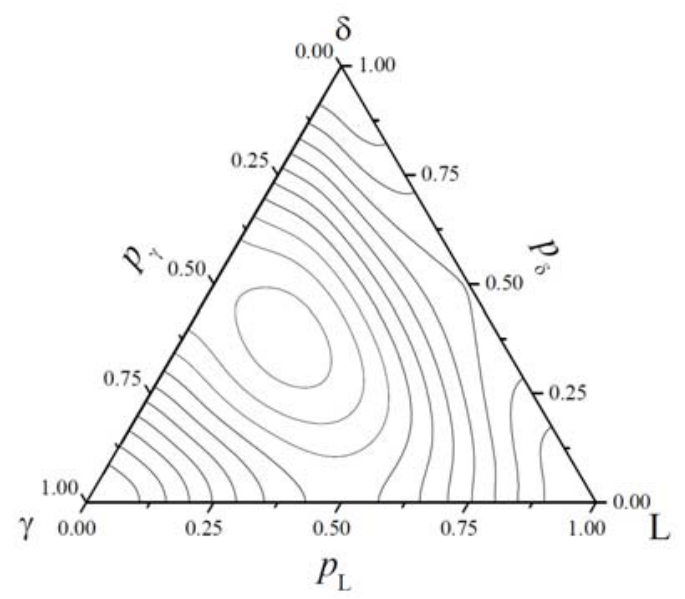

(c)

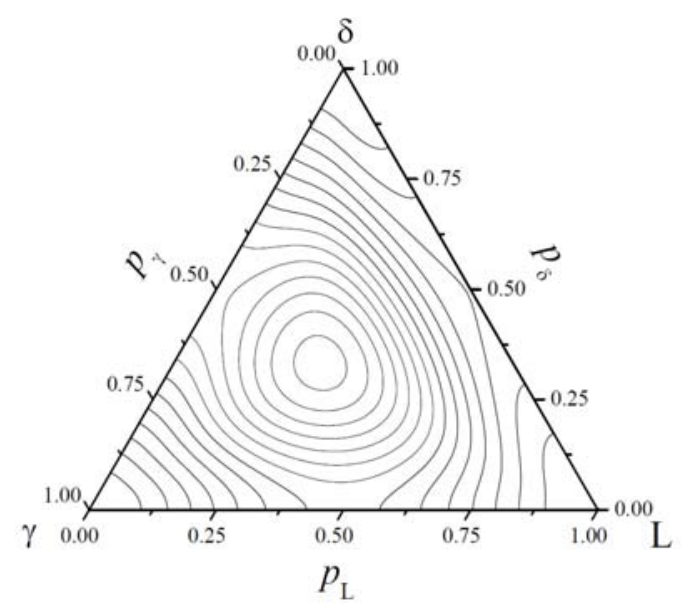

(b)

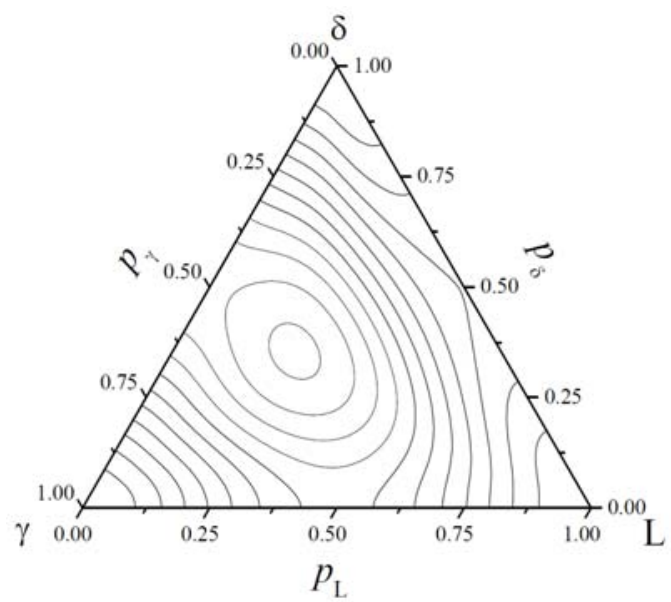

Fig. S3. Potential surfaces of $f_{\text {pot }}$ in the Gibbs simplex calculated for $n=2.0$ and (a) $\omega_{t r i}=2 \omega^{*}$, (b) $\omega_{t r i}=3 \omega^{*}$ and (c) $\omega_{t r i}=7 \omega^{*}$. In each figure, the interval of the contour lines is $\Delta f_{\text {pot }}=5 \times 10^{5} \mathrm{~J} / \mathrm{m}^{3}$.

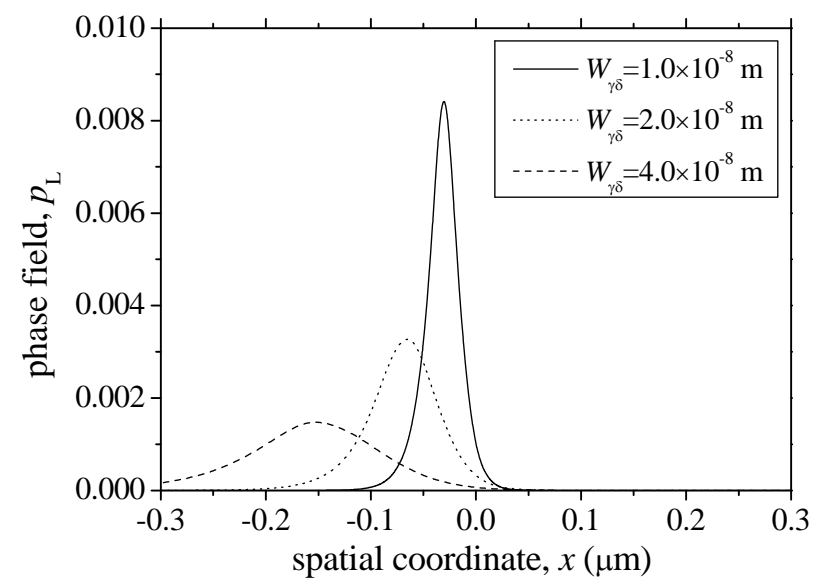

Fig. S4. Spatial profiles of $p_{\mathrm{L}}$ across $\gamma-\delta$ interface along $x$ direction calculated without the additional potential for different values of $W_{\gamma \delta}$. The origin of the horizontal axis corresponds to the center position of $\gamma-\delta$ interface. 


\section{B. Difference in convergence behavior between the cases of equal and unequal interfacial energies}

In the early works for the quantitative phase-field modeling [11, 13, 20, 21], the effects of thin-interface corrections were discussed based on the convergence curve with respect to $W / d_{0}$ where $d_{0}$ is the chemical capillary length. In the present model, the chemical capillary length depends on the type of interface as expressed by $d_{0, i j}$. $W_{i j} / d_{0, i j}$ is calculated in this model as follows,

$$
\frac{W_{i j}}{d_{0, i j}}=\frac{3 \sqrt{2} R T_{m, i j}}{v_{m} \omega_{i j}}\left(1-k_{i j}\right)\left(c_{j, e}^{i}-c_{i, e}^{j}\right) .
$$

When the simulations for the unequal interfacial energy and the conditions $\Delta T=10 \mathrm{~K}$ and $W_{\gamma \delta} \leq 3.0 \times 10^{-8} \mathrm{~m}$ are considered (Fig. 4), the values of $W_{i j} / d_{0, i j}$ are always less than 10.2 for the $\delta$-L interface, 2.9 for the $\gamma$-L interface and 0.36 for the $\gamma$ - $\delta$ interface. Also, in the calculations for $\Delta T=5 \mathrm{~K}$ and $W_{\gamma \delta} \leq 4.0 \times 10^{-8} \mathrm{~m}$, the values of $W / d_{0}$ are less than 12.3 for the $\delta$-L interface, 3.4 for the $\gamma$-L interface and 0.50 for the $\gamma$ - $\delta$ interface. Judging from the convergence curves reported in the early works $[11,13,21]$, these values of $W_{i j} / d_{0, i j}$ corresponds to relatively small values where one can generally expect the well converged result. In fact, almost the constant value is observed in Fig. 4. However, the results for the equal interfacial energies seem to be not converged even for the smallest value of $W_{\gamma \delta}$ used in this study. This point is discussed here.

Figure 2 shows that the convergence of the results for the equal interfacial energy is slower than that for the unequal interfacial energy. The steady state shape of the interfaces near the triple junction at $\Delta T=10 \mathrm{~K}$ is shown in Fig. S5(a). These are the results for the equal interfacial energy. One sees that the steady state shape is not well converged in the range of $W_{\gamma \delta}$ tested here. The steady state shape for the equal interfacial energy is compared with that for the unequal interfacial energy in Fig. S5(b). The curvatures of $\gamma-\delta$ and $\gamma$-L interfaces near the junction in the former case are larger than those in the latter case, as is expected from the balance between the interfacial energies near the junction (Young's rule). The velocity of growing $\gamma$ phase (peritectic reaction rate) for the equal interfacial energy is much higher than the one for unequal interfacial energy as is seen in Fig. 2. Therefore, the peritectic reaction for the equal interfacial energy involves the relatively large curvatures and the high migration velocities of the interfaces. The anomalous interface effect becomes significant in this situation, as is realized from the asymptotic analysis [13, 21]. This should be the reason why the convergence of the results for the equal interfacial energy is slow. 
(a)

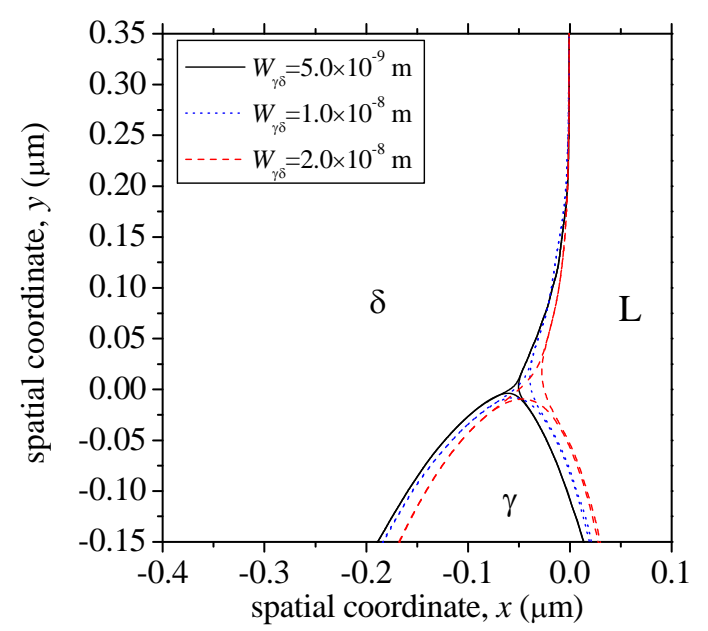

(b)

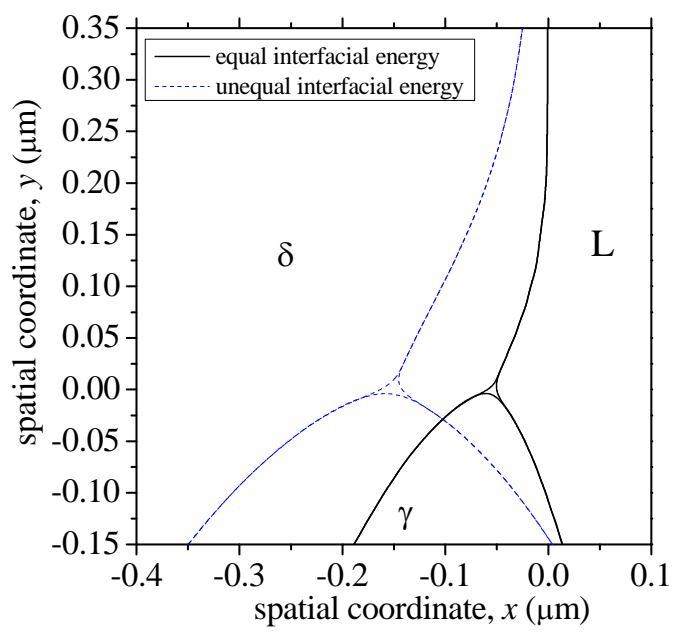

Fig. S5. (a) Steady state shapes of the interfaces near the triple junction at $\Delta T=10 \mathrm{~K}$ for the case of equal interfacial energy. (b) Comparison between the steady state shapes near the triple junction for equal interfacial energy (solid line) and unequal interfacial energy (dashed line). The interface thickness $W_{\gamma \delta}$ is $5.0 \times 10^{-9} \mathrm{~m}$ for the former case and $1.0 \times 10^{-8} \mathrm{~m}$ for the latter case. In each figure, the origin of $y$ axis is the position of the triple junction and the origin of $x$ axis corresponds to the initial position of L- $\delta$ interface. 